\title{
SOME GENERAL GRADIENT ESTIMATES FOR TWO NONLINEAR PARABOLIC EQUATIONS ALONG RICCI FLOW
}

\author{
Wen Wang, Hui Zhou, Rulong Xie And Songting Yin
}

\begin{abstract}
In this paper, by maximum principle and cutoff function, we investigate gradient estimates for positive solutions to two nonlinear parabolic equations along the Ricci flow. As applications, the related Harnack inequalities for positive solutions to the nonlinear parabolic equations along the Ricci flow are derived. These results can be regarded as generalizations of the results of Li-Yau, J. Y. Li, Hamilton and $\mathrm{Li}-\mathrm{Xu}$ to a more general nonlinear parabolic equation along the Ricci flow. Our results also improve the estimates of S. P. Liu, J. Sun and Y. Y. Yang to the nonlinear parabolic equation along the Ricci flow.
\end{abstract}

Mathematics subject classification (2010): 58J35, 35K05, 53C21.

Keywords and phrases: Gradient estimate, nonlinear parabolic equation, heat equation, Ricci flow, Harnack inequality.

\section{REFERENCES}

[1] Abimbola Abolarinwa, Gradient estimates for a nonlinear elliptic equation on complete noncompact Riemannian manifold, Journal of Mathematicaln Inequalities 12(2), (2018), 391-402.

[2] M. Bailesteanu, X. D. CaO, A. Pulemotov, Gradient estimates for the heat equation under the Ricci flow, J. Funct. Anal., 258 (2010), 3517-3542.

[3] E. CALABI, An extension of E. Hopf's maximum principle with an application to Riemannian geometry, Duke Math. J. 25 (1958), 45-56, MR 19,1056e Zbl 0079.11801.

[4] H. CAO, M. ZHU, Aronson-Bénilan estimates for the porous medium equation under the Ricci flow, Journal De Mathématiques Pures Et Appliquées, 2015, 104(4):90-94.

[5] X. D. CAO, B. F. Ljungberg, B. W. LiU, Differential Harnack estimates for a nonlinear heat equation, J. Funct. Anal., (2103): 1-19.

[6] D. G. CHEn, C.W. XIONG, Gradient estimates for doubly nonlinear diffusion equations, Nonlinear Anal. 112 (2015), 156-164.

[7] B. Chow And D. Knopf, The Ricci flow: An introduction, mathematical Surveys and Monographs 110, American Society, Providence, RI, 2004, MR 2005e:53101 Zbl 1086.53085.

[8] R. S. Hamilton, A matrix Harnack estimates for the heat equation, Comm. Anal. Geom., 1 (1993), $113-126$.

[9] R. S. Hamilton, Three manifolds with positive Ricci curvature, J. Differential Geom. 17:2 (1982): 255-306, MR 84a:53050 Zbl 0504.53034.

[10] R. S. Hamilton, The formation of singularities in the Ricci flow, pp. 7-136 in Surveys in differential geometry, II (Cambridge, MA, 1993), edited by S. T. Yau, International, Cambridge, MA, 1995., MR 97e:53075 Zbl 0867.53030.

[11] G. Y. HuAng, Z. J. HuAng, H. Z. LI, Gradient estimates and differential Harnack inequalities for a nonlinear parabolic equation on Riemannian manifolds, Ann Glob Anal Geom, (2013) 43: 209-232.

[12] S. KUANG, Q. S. ZHANG, A gradient estimate for all positive solutions of the conjugate heat equation under Ricci flow, J. Funct. Anal. 255:4 (2008), 1008-1023, MR 2433960 Zbl 1146.58017.

[13] H. LI, H. BAI, G. ZHANG, Hamilton's gradient estimates for fast diffusion equation under the Ricci flow, J. Math. Anal. Appl., 444 (2016) 1372-1379.

[14] P. LI, S. T. YAU, On the parabolic kernel of the Schrödinger operator, Acta Math., 156 (1986), 153-201, MR 87f:58156. 
[15] J. Y. LI, Gradient estimates and Harnack inequalities for nonlinear parabolic and nonlinear elliptic equations on Riemannian manifolds, J. Funct. Anal., 100 (1991), 233-256.

[16] J. Y. LI, Gradient estimate for the heat kernel of a complete Riemannian manifold and its applications, J. Funct. Anal., 97:2 (1991), 293-310, MR 92f:58174 Zbl 0724.58064.

[17] J. LI AND X. XU, Differential Harnack inequalities on Riemannian manifolds I: Linear heat equation, Adv. in Math., 226 (2011), 4456-4491.

[18] S. P. LIU, Gradient estimates for solutions of the heat equation under flow, Pacific J. of Math., 243 (1), (2009), 165-179., MR 2010g:53122 Zbl 1180.58017.

[19] Y. LI, X. R. ZHU, Harnack estimates for a heat-type equation under the Ricci flow, J. Differential Equations 260 (2016), 3270-3301.

[20] P. Lu, L. Ni, J. L. VÁZqueZ And C. Villani, Local Aronson-Bénilan estimates and entropy formulae for porous medium and fast diffusion equations on manifolds, J. Math. Pures Appl. 91 (2009), $1-19$.

[21] L. MA, Gradient estimate for a sample elliptic equation on non-compact Riemannian manifolds, J. Funct. Anal., 241 (2006), 374-382, MR 2264255(2007e:53034).

[22] G. Perelman, The entropy formula for the Ricci flow and its geometric applications, preprint, 2002, arXiv math.DG/0211159.

[23] G. Perelman, Ricci flow with surgery on three manifolds, preprint, 2003, arXiv math.DG/0203109.

[24] W. X. SHI, Deforming the metric on complete Riemannian manifolds, J. Differential Geom., 30: 1 (1989), 223-301, MR 90i:58202 Zbl 6076.53044.

[25] B. H. Song, The Harnack Estimate for a Nonlinear Parabolic Equation under the Ricci Flow, Acta Mathematica Sinica, English Series 27(10),(2011): 1935C1940 DOI: 10.1007/s10114-011-0074-z.

[26] J. Sun, Gradient estimates for positive solutions of the heat equation under geometric flow, Pacific J. Math. 253, 489-510 (2011).

[27] H. J. Sun, Higher Eigenvalue Estimates on Riemannian Manifolds with Ricci Curvature Bounded Below, Acta Math. Sinica (Chin. Ser.) 49 (2006), 3, 539-548.

[28] W. WANG, P. Zhang, Some Gradient Estimates and Harnack Inequalities for Nonlinear Parabolic Equations on Riemannian Manifolds, Mathematische Nachrichten, (2017) 290,11-12,1905-1917.

[29] J. Y. WU, Li-Yau type estimates for a nonlinear parabolic equation on complete manifolds, J. Math. Anal. Appl., 369 (2010), 400-407.

[30] Y. Y. YANG, Gradient estimate for a nonlinear parabolic equation on Riemannian manifold, Proc. Amer. Math. Soc. 136 (2008),4095-4102.

[31] Y. Y. YANG, Gradient estimates for the equation $\Delta u+c u^{\alpha}=0$ on Riemannian manifolds, Acta. Math. Sin. 26(B), 1177-1182, (2010).

[32] L. ZHAO, S. FANG, Gradient estimates foe a nonlinear lichenrowicz equation under generalize geometric flow on complete noncompact manifolds, Pacific J. Math. 285 (1) (2016):243-256.

[33] X. B. ZHU, Gradient estimates and Liouville theorems for nonlinear parabolic equations on noncompact Riemannian manifolds, Nonlinear Analysis, 74 (2011), 5141-5146. 